\title{
Wandlungsmotor SARS-CoV-2
}

\section{Wegweiser zum Gesundheitsstandort der Zukunft}

Als größte Gesundheitskrise in der Geschichte stellt SARS-CoV-2-Pandemie die Welt auf eine harte Probe. Gleichzeitig deckt sie die Schwachstellen des globalen Gesundheitssystems auf und ebnet den Weg für neue Entwicklungen. Auch für Österreich und Europa wird sichtbar, wo es Chancen und Entwicklungspotenzial gibt. Dies wurde bei einer Veranstaltung der PHARMIG ACADEMY im November in Wien diskutiert.

\section{Digitale Kommunikation boomt} „Ob virtuelle Ärztekonsultationen, digitale Diagnostik oder IT-Algorithmen im Forschungsbereich, die Pandemie hat Entwicklungen wie der Digitalisierung einen ordentlichen Schub verpasst", sagt Prof. Dr. Robin Rumler, Präsident der PHARMIG ACADEMY und Vizepräsident der PHARMIG, im Rahmen der virtuellen Veranstaltung HEALTH CARE SYMPOSIUM der PHARMIG ACADEMY. Durch den notwendig gewordenen Einsatz digitaler Kommunikationswerkzeuge können beispielsweise Health Care Professionals ihren gegenseitigen Kontakt nun viel flexibler, effizienter, individueller und therapiezentrierter gestalten. Davon profitiere der
Kontakt zwischen Ärzteschaft, medizinischem Fachpersonal und dem Außendienst pharmazeutischer Unternehmen. „Denn dadurch kann das Wissen zur Wirkungsweise von Therapien schneller rückgemeldet werden", so Rumler.

\section{Forschung und Zulassung beschleunigt}

Bisher habe die Pandemie laut Rumler in hohem Maße dazu beigetragen, das Forschungstempo auf der Suche nach einem Impfstoff gegen SARS-CoV-2 zu beschleunigen. Das zuständige Bundesamt für Sicherheit im Gesundheitswesen (BASG) ist in den gesamten Zulassungsprozess von Arzneimitteln involviert und hat die Aufgabe, den Nutzen sowie das Risiko neu zuzulassender Medikamente zu prüfen. Auch wenn die Pandemie gezeigt hat, dass Zulassungsprozesse schneller verlaufen können, braucht es unabhängig vom öffentlichen Diskurs valide Forschungsdaten, um Sicherheit für jene zu garantieren, die den Impfstoff dann verabreicht bekommen. Für Covid-19 Impfstoffe gibt es ein beschleunigtes Zulassungsverfahren und ein so genanntes „Rolling Review“-Verfahren. Das bedeutet, dass die behördliche Begutachtung bereits parallel bei den letzten Entwicklungsschritten beginnt.

Anschließend erfolgt ein kurzes Zulassungsverfahren. Beide erfolgen

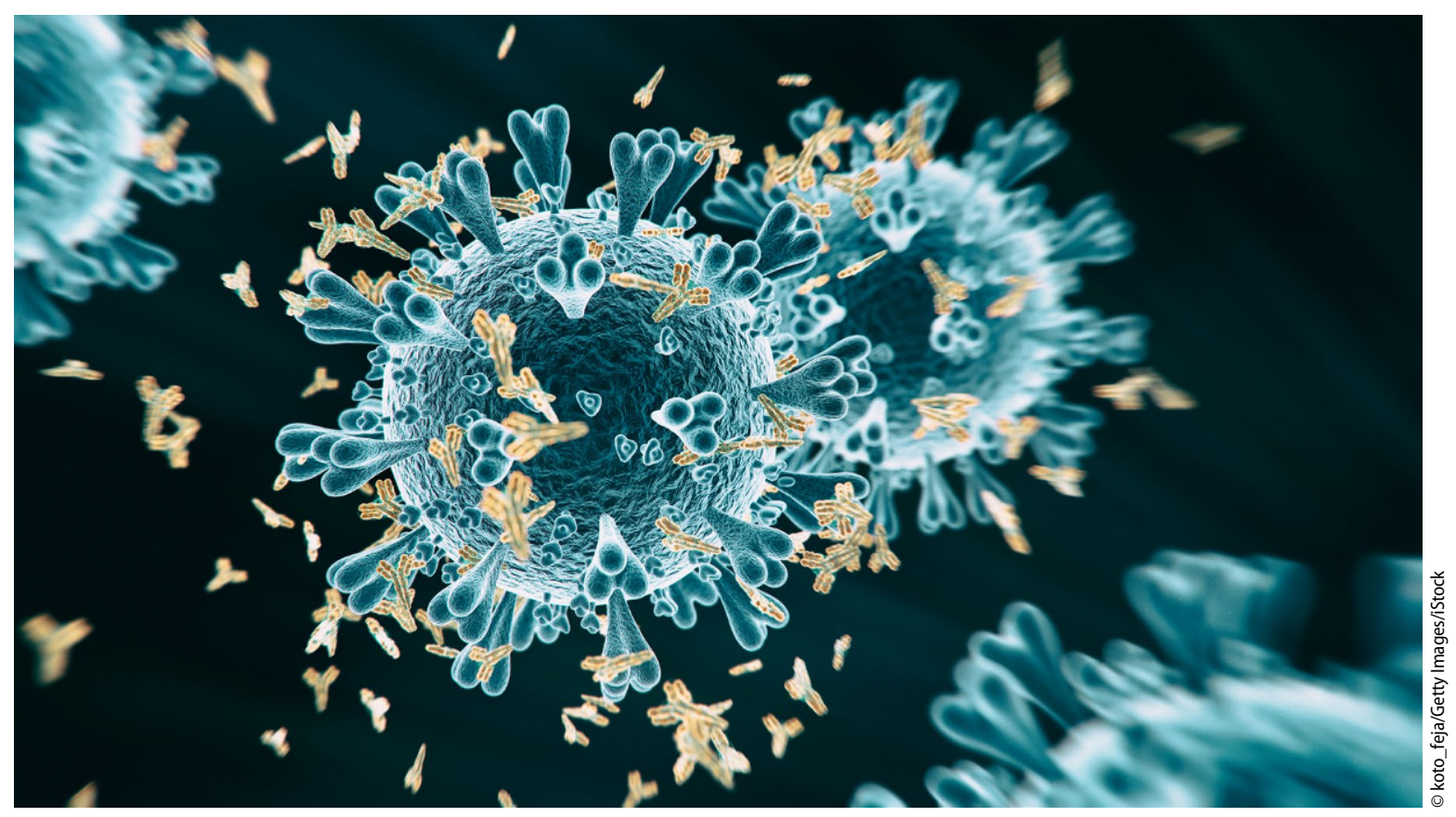


ohne Abstriche in Qualität und Umfang der Begutachtung und sollen rasch eine Zulassung wirksamer und sicherer Impfstoffe gegen Covid-19 ermöglichen.

\section{Mehr klinische Studien in Österreich}

Hinsichtlich der Forschungsaktivitäten verdeutlicht die Pandemie, dass Österreich nach wie vor nicht zu jenen Ländern zählt, in denen viele Studien zur Arzneimittel-Entwicklung laufen. Die Bevölkerung ist hierzulande sehr zurückhaltend, wenn es um die Teilnahme an klinischen Forschungsprojekten geht. Um seinen Wirkstoffkandidaten gegen SARS-CoV-2 im Zuge der klinischen Forschungsphase II zu prüfen, musste Forscher Prof. Dr. Josef Penninger sogar in Russland Patientinnen und Patienten mit fortgeschrittenem COVID-19-Verlauf rekrutieren. Aussagekräftige Ergebnisse seiner Studie sollen bis zum Jahresende vorliegen. Hier verliert Österreich Knowhow, vor allem was die Entwicklung von und den Zugang zu hochinnovativen Medikamenten betrifft. Dazu sagt PHARMIG-Präsident Philipp von Lattorff: „Klinische Studien erfüllen eine gesellschaftlich wertvolle Rolle. Es braucht konkrete Maßnahmen der Politik, um Forschungseinrichtungen zu fördern und um Voraussetzungen zu schaffen, dass mehr klinische Studien in Österreich durchgeführt werden. Denn ohne klinische Prüfungen gibt es keine Innovation und somit auch keine Verbesserung der medizinischen Versorgung von Patientinnen und $\mathrm{Pa}-$ tienten."

Quelle: Das HEALTH CARE SYMPOSIUM der PHARMIG

ACADEMY, moderiert von "Die Presse“-

Redakteur Köksal Baltaci, fand am

2. November 2020 virtuell mit 200 Teil-

nehmerinnen und Teilnehmern statt.

OTS, 11.11.2020 sitzender des EU-Steering Boards für die Beschaffung des Impfstoffes gegen SARS-CoV-2 sowie Sonderbeauftragter für Gesundheit im österreichischen Bundesministerium für Gesundheit. Laut Auer sei die Position der EU-Länder in Gesundheits- und Versorgungsfragen seit der Pandemie geeinter als zuvor, vor allem, wenn es um die Zusammenarbeit mit der pharmazeutischen Industrie gehe. Für die Arzneimittelversorgung der Zukunft brauche es laut Auer, aber auch nach Meinung des PHARMIG-Präsidenten von Lattorff, eine gesamteuropäische Strategie statt nationale Einzellösungen. Dieses gemeinsame Vorgehen dürfe keinesfalls auf den aktuellen Bedarf bei SARS-CoV-2-Impfstoffen reduziert bleiben.

\section{Paradigmenwechsel tut not}

Die Auswirkungen der Pandemie decken laut Auer auch die hohe Wirkstoffabhängigkeit Europas von Asien auf, die in derVergangenheit durch das niedrige Preisniveau bei Arzneimitteln genährt wurde. Laut Auer brauche es hier einen Paradigmenwechsel, damit der Erfolg der Vergangenheit nicht zum Misserfolg der Gegenwart werde. „Möchte die EU langfristig die Produktionssicherheit für zentrale Wirkstoffe innehaben, wird man nicht umhin kommen, sich darüber Gedanken zu machen, was die daraus resultierende Versorgungssicherheit kostet und diesen Aspekt in Zukunft bei der Preiskalkulation zu berücksichtigen“, sagt Auer. Andenfalls sei fraglich, wie Standortpolitik verbessert werden könnte.

PHARMIG-Präsident von Lattorff zählt einige Punkte zur Standortverbesserung auf: „Steuererleichterungen, ein klares Bekenntnis zu mehr Forschung oder konkrete Maßnahmen, um die Produktion in Österreich weiter attraktiv zu machen - das alles sind entscheidende Faktoren, die zur Spitzenleistung der pharmazeutischen Industrie in Österreich beitragen und zum Vorteil der Gesamtwirtschaft beachtet werden sollten. Die Weichen hierfür können aber nur gemeinsam von allen Playern am Standort gestellt werden."

\section{Niederschwellige Information für die Bevölkerung}

Diesem Ruf nach einem gemeinsamen Vorgehen schließt sich auch Wolfgang Katzian, Präsident des Österreichischen Gewerkschaftsbundes, an. Die Pandemie habe die Grenzen der Leistungsfähigkeit des österreichischen Gesundheitssystems verdeutlicht. Auch brauche die Bevölkerung angesichts der Erforschung eines SARS-CoV-2-Impfstoffs mehr niederschwellig zugängliche Informationen, damit die Bedürfnisse und Beweggründe der pharmazeutischen Industrie öffentlich nachvollziehbar seien. Nur so könne dafür gesorgt werden, Durchimpfungsraten zu erhöhen und langfristig ein solidarisches und gemeinschaftlich finanziertes Gesundheitssystem zu etablieren. Daher müsse alles unternommen werden, um Forschung und Entwicklung in Österreich sicher zu stellen. Das habe die Pandemie deutlich gezeigt.

\section{Weitere Informationen:}

www.pharmig.at

Hinweis des Verlags. Der Verlag bleibt in Hinblick auf geografische Zuordnungen und Gebietsbezeichnungen in veröffentlichten Karten und Institutsadressen neutral.

Paediatr. Paedolog. 2020 · 55:327-328 https://doi.org/10.1007/s00608020-00852-8

(c) Springer-Verlag GmbH Austria, ein Teil von Springer Nature 2020 